\title{
Machine Learning: Prospects, Opportunities and Benefits to the Greek Railways
}

\author{
I. Kalathas \\ Dept. of Industrial Design \\ and Production \\ Engineering University of \\ West Attica \\ Athens, Greece
}

\author{
M. Papoutsidakis \\ Dept. of Industrial Design \\ and Production \\ Engineering University of \\ West Attica \\ Athens, Greece
}

\author{
D. Piromalis \\ Dept. of Industrial Design \\ and Production \\ Engineering University of \\ West Attica \\ Athens, Greece
}

\author{
L. Katsinoulas \\ Dept. of Industrial Design \\ and Production \\ Engineering University of \\ West Attica \\ Athens, Greece
}

\begin{abstract}
One of the areas of Artificial Intelligence that inspired a keen interest on scientists, and not only, from the very beginning of its foundation in the 1980 s, was the subject of Machine Learning.

Machine Learning comprises a field of study quite extensive and of great interest which attracted many researchers. Its objective consists of the construction of programs capable of adapting automatically their operation in order to improve their performance, through the experience gained during their execution. It is about improving computer skills in specific areas, as well as improving human-computer connection and interaction. It can be applied in personal computers, everyday life and in key areas of life such as education, finance and transport.
\end{abstract}

Implementing, planning and designing transport infrastructures and investments is a difficult task, whether it concerns an intergraded intervention or an effort for local improvement. The main advantage of the use of a railway network, is the transportation from one place to another within a minimum time spent. Rail transport infrastructure is part of the development field and is in line with the development of each region.

Transport companies are very important to the economy and progress of each country, since they contribute significantly to all the services provided by the state. The services provided to the citizen must constantly be improved and upgraded with the primary objective of the safe transport of passengers and goods.

This paper presents the technology of mechanical learning and its contribution to transport, especially on railway networks and railway undertakings. There is a theoretical introduction to mechanical learning and a brief review of emerging technology with a historical retrospection on the major milestones of its course. At the same time, its basic features, methods and algorithms are examined.

Innovative applications used in transport, and in particular on railway networks are included. This paper highlights the problems of the Greek railways and records the needs and requirements of the transport companies. Finally, the conclusions and incentives of railway companies for the creation of engineering learning applications to be used as a tool for the development and continuous improvement of the quality of the services provided are presented.

\section{Keywords}

Machine learning, application, railway, rolling stock, maintenance, data, knowledge mining

\section{INTRODUCTION}

Learning is one of the fundamental attributes of man's intelligent behavior. It is a process that leads to a constant change in a person's behavior that results from experience or exercise. The acquisition and change of knowledge, skills, strategies, beliefs, attitudes and various behaviors, that is, the process in which the individual's cognitive potential changes, results from the diverse experiences the individual processes[1][2]. It is typically said that learning occurs when there is a clear change in behavior, resulting from a process other than maturing. Learning can be interpreted as a series of events that lead to behavioral change and which are causatively connected with change.

In relation to the human ability to learn, philosophers ask the question: "How can an inductive reasoning that leads to learning be evaluated in terms of its correctness?" Accordingly, psychologists ask: "How does the brain store the results of the learning process, that is, the mental models and patterns?" In the field of Artificial Intelligence, they simply ask: "How can a machine create new models and learning patterns from concrete examples and how reliable are these models in practice?"[3]

Machine learning is called the sector of artificial intelligence which deals with the study or the construction of models capable of "learning" from a large number of data. Machine learning is a field of computer science and belongs to the wider area of artificial intelligence. Machine learning algorithms can learn from data and make predictions for new data that have not been predicted. The term is relatively new, the idea though derived from the field of statistics that deals with forecasting and mathematical optimization. Machine learning comes to help where traditional programming faces obstacles.

Machine learning is a method used to devising complex models and algorithms that lead to prediction. Analytical models allow researchers, data scientists, engineers to make trusted decisions and results[4]. Essentially, the field of machine learning deals with all those processes that attribute to computers, more complex capabilities than performing simple, prescribed and scheduled processes.

It finds applications in the economy, medicine, education, transport and is directly related to improving computer capabilities, progress and business development.

In recent years, major developments in global economic growth and the emergence of new business-related technologies have raised new challenges for data and information management as a means of computing, since they have accumulated a huge multitude of data. It is thus, 
commonly accepted, that any form of organization or modern business today is highly dependent on technology. The use of new technologies gives all companies a significant competitive advantage, while the lack of new technologies can be fatal to a business and even end it[5][6].

Transport companies are increasingly related to the efficient use of information as well as to the systems that supply it, which are the information systems. Railway companies use engineering learning applications to optimize the use of data and draw useful conclusions so that the company has the best information in the planning of its strategic moves and achieve the overriding goal of passenger and trains' safety.

The use of rail, for the transport of goods and human resources, is necessary to maintain and develop. One of the most important reasons is that rail transport is one of the most economical ways of transport. Wherever there is developed mass transport by rail, there is a strong economic activity such as businesses, employment, easy access to markets, trade development, comfortable and fast transportation of people and goods, tourism development[7]. In general, rail transport is a key instrument to growth. The accessibility of people to work, health, education and other social activities is of essential importance to the well-being of people in rural and urban areas.

\section{MACHINE LEARNING}

\subsection{Definition of Machine Learning}

Machine learning is defined as the search of informational systems which can automatically improve, with the use of algorithms and methods, the efficiency of a machine to perform "intelligent" tasks. It is the phenomenon of a system that improves its efficiency during a particular task and allows on the software applications to become more precise on the prediction of results without being explicitly programmed. The science of machine learning creates computers which learn to act as people and improve their learning on their own as the time goes by, supplying their data and information with the form of comments or real interactions.

\subsection{Chronology}

As scientific venture, the machine learning was developed by the search of artificial intelligence. From the primary period of the search in the field of artificial intelligence on academic level, the issue of construction of machines capable of learning data interested researchers. The ideas of machine learning have a long background and are based on mathematics from hundreds of years ago and on huge developments in the field of information the last 70 years. The actual creation took place near the 1700 and was based on the probability theory of Bayes. The following remarkable scientific accomplishments which are utilized on the applications of machine learning first appeared in the middle of the 20th century.

The conception of artificial intelligence belongs to the writer Walter Pitts and the neuroscientist Warren McCulloch who created the first mathematical model of a neural network in 1943. They published their huge work "A Logical Calculus of the Ideas immanent in nervous activity", suggesting a combination of mathematics and algorithms that target on imitating the process of human thoughts.

In 1950, Alan Turing published the research "Computing Machinery and Intelligence" on which he wondered: "Can machines think?" - A question that we still struggle to answer. He suggested a test which he named "imitation game". In order to define if a computer is intelligent, he asked from a person to make a distinction between a human and a computer when the person interacted with each one of them, through typed messages.

The pillar of the modern artificial intelligence is attributed to Arthur Samuel, who taught a computer to play checkers by imitating the movements described on the book "Lee's Guide to Checkers". The term "machine learning" was initially suggested by Arthur Samuel in 1959, and is referred on computer systems that have got the ability to learn from large quantities of previous tasks and data without being programmed by the use of algorithms [8].

At the beginning of 1960 the blossom of machine learning practically starts through artificial intelligence. The logical and based on knowledge systems like the logical programming and the experts' systems, along with the development of statistic data and theoretical reflective approaches create excellent techniques and methods algorithms of machine learning - that still comprise the most productive approaches to many problems with most significant methods the Decision Trees, the Cluster Analysis and the Genetic Algorithms. Later on, an increasing emphasis on approaches based on logical knowledge caused a rift between Artificial Intelligence and Machine Learning.

The Machine Learning, was reorganized as a separate field, which started to flourish during the ' 90 s. The attention was relocated from the symbolic approaches inherited by the Artificial Intelligence aiming to face the solution of practical problems to emphasizing on statistic methods and models and the theories of probabilities.

In 1997 Tom M. Mitchel suggested a more official definition on machine learning which is widely used: " A computer programme is said to learn from experience $E$ with respect to some class of tasks $\mathrm{T}$ and performance measure $\mathrm{P}$ if each performance at tasks in $\mathrm{T}$, as measured by $\mathrm{P}$, improves with experience E." During the 2000 s, researchers with the improvement of computers, have gradually renewed the interest and consequently the machine learning infiltrates into a wide spectrum of services and applications [9].

Today the machine learning consists of a field of learning wide enough and with great interest. It is connected with the improvement of computer possibilities the progress and improvement of businesses but also the reinforcement of the relation between man and computer. It applies on finance, medicine, education, everyday life and the personal computers and many more that are constantly increasing.

\subsection{Types of Machine Learning}

The field of Machine Learning develops three methods of learning in relation to the ways that people learn and the cluster according to the type of data and the results expected: supervised learning, unsupervised learning and reinforcement learning [10].

- Supervised learning is the process of an algorithm building a function which depicts input of marked data to a known output, having as a final target to apply a model of prediction to new data which have not been seen. It is used in problems of classification, prediction and interpretation.

- Unsupervised learning is the process of an algorithm building a model for a total of inputs in the form of observation without knowing the desired outputs. It is used in problems of association 
analysis and clustering.

- Reinforcement learning of an algorithm learning a strategy of acts through instant interaction with the environment. It is mainly used to face problems of planning and in dynamic environments like selfdriving cars or real-time games [11].

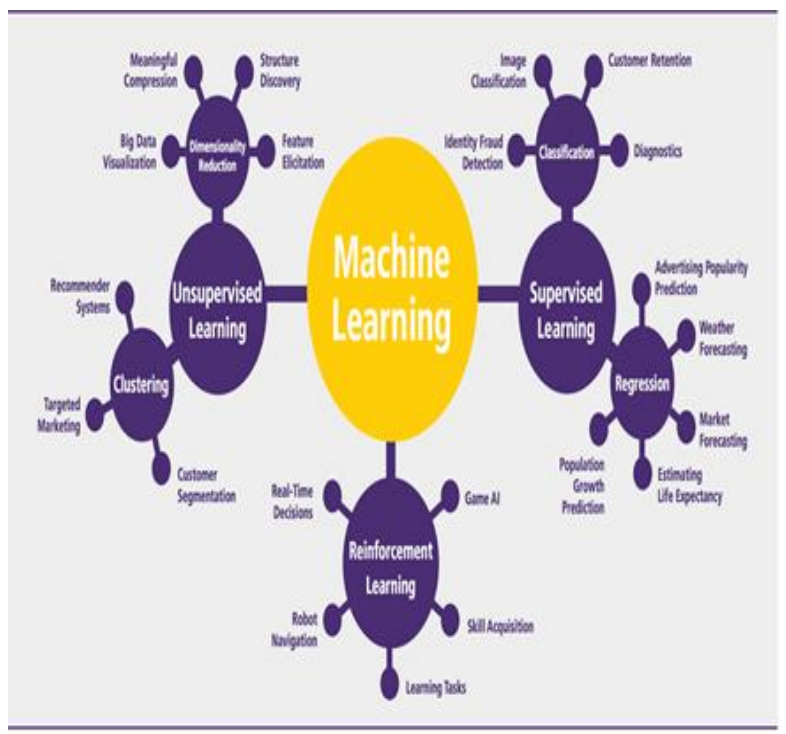

Fig 1: Types and Categories of problems in Machine Learning

\subsection{Categories of problems in Machine Learning}

Depending on the type of prediction - supervision that has to be answered through Machine Learning the categories of problems are the following:

- Classification: The classifier usually separates the data in categories and tries to predict new data into supervised environment. The machine has to produce a model, which will respond to the data in one or more classified categories.

- Clustering: A number of inputs is going to be separated into groups. Contrary to classification the groups are not known beforehand, rendering the separation a typical task of unsupervised learning.

- Retrogression: A problem on supervision, the output here is constant and undiscernible, though.

- Density estimation: Finding of the allocation of the input data somewhere.

- Dimensions decrease: Simplification of inputs into lower dimensions. It is required when the data is more complex with many variables. The data are simplified and classified in a space of less dimensions.

\subsection{Algorithms of Machine Learning}

The Machine Learning consists of a great variety of algorithms which can be separated into categories depending on the kind of learning, the technique followed and the problems that need to be solved.

- Decision trees: The approach of an unknown distinct function is attempted. The model of prediction is illustrated into a decision tree which classifies its observations on its branches. The main feature is placed in the root of the tree. The main advantage is the ease of comprehension of the prediction process even from non-experts. As far as efficiency is concerned, it is highly effective.

- Artificial Neural Networks: They are inspired by the structure and the functions of the biological neurons, the artificial neural networks build their input observations in the form of joins. They consist of a total of complicated interconnected simple units, arranged usually in layers. They are used for the modeling complicated relations between the input-output and the model recognition.

- Logistic Regression: From the previous linear statistical models of prediction (1958), logistic regression provides binary classification with one or more variables. One of the main advantages is the interpretation of the weight of each variable in the final decision of classification.

- Nearest neighbor: this method is one of the most popular, oldest and the most reliable methods of classification. Depending on the class of the nearest neighbours that an observation contains, it is classified by majority. The parameter that changes allows us to examine the number of neighbours to improve the result. It has been applied in the field of statistics, the modelling and the recognition of prototypes and it is lately used in the field of machine learning.

- K-means: is an algorithm of clustering characteristics in an arithmetical form. The number $\mathrm{k}$ of the teams is defined before the execution of the algorithm. It does not guarantee the unique clustering result of the neighbor data points. One of its main advantages is its speed [12].

Clearly there are other algorithms as well, with variations in architecture and methodology. The more concrete and effective models are usually combinatorial with the use of aiding techniques, of course.

\subsection{Stages of realization of Machine \\ Learning}

The first stage of planning of a system of machine learning is the definition of the knowledge that it will be used during its training. For every problem that has to be solved there is a proper way of learning for which there is at least an appropriate algorithm that can be applied. This procedure is called training. The allocation of examples, from which the knowledge will be drawn by the system of machine learning, is of great importance for the quality of the training set.

The next phase is the processing of data for the attestation of the new knowledge produced. The attestation is a way that the system (usually by the algorithm itself) and with a selection of parameters obtains knowledge targeting its more productive function.

Then a control takes place for the capacity of generalization of the systems' data and afterwards the assessment of the progress of the models takes place through the user and the knowledge it obtains. Finally, the new knowledge is given to be used in applications to which it is necessary in order for real problems to be solved, otherwise it feedbacks the model with new data [13]. 


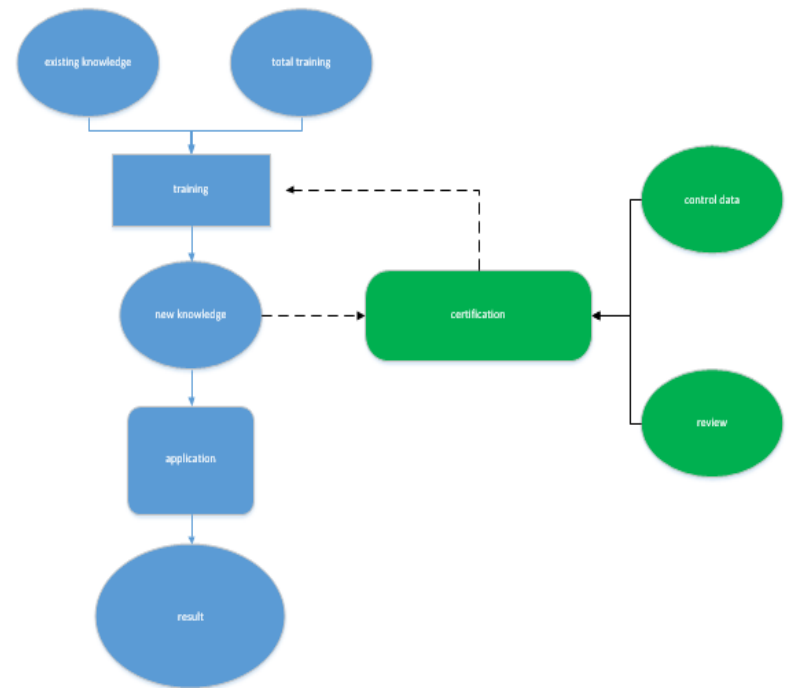

Fig 2: Machine learning stages

\section{APPLICATIONS OF MACHINE LEARNING}

Machine learning is behind many applications that we daily use, from automatic translation, the automatic recognition of faces on Facebook, the visual recognition of characters and speech, the tracing of unwanted mail (spam filtering) and the search engines. Generally in the last decade machine learning has offered to humanity a vast number of innovative products and systems [14]. A field which arises a constant increasing interest for the use of methods of machine learning is transportation. The changes in the transport sector are impressive, minimizing the accidents, significant decrease in functional cost and improving the level of the services offered. With the use of machine learning the habits of citizens' transportation change, as well as they ways the cities are organized and developed.

\subsection{Car industry}

In the sector of car industry the competitiveness is increasing according to the data analysis. The applications of machine learning cater for the progress of technology from the motor vehicles to the financial transactions. From the tracking of a problem and the diagnosis to digital marketing of car industries.

Researchers of the federal research centre of science and technology of the USA Berkeley Lab have demonstrated that machine learning can help in the increase of sustainability of the car industry, as far as the traffic management is concerned as well as the energy consumption. They use the machine learning to train autonomous vehicles in order to drive in a way that improves the traffic flow and decrease the energy consumption at the same time. They also apply algorithms of machine learning to analyze satellite pictures which in connection with information of motion from mobile phones and data gathered from environmental sensors improve the predictions for the quality of fumes [15].

\subsection{Shipping}

In the sector of shipping machine learning makes ships safer, easier to use and more effective, offering to the crew the capacity of better perception of the environment around the ship. The Rolls-Royce Company, has signed and an agreement with Google to develop further its "intelligent" systems which render the present vessels safer and they are necessary in order to make autonomous ship a reality.
According to this, Rolls-Royce can use the Cloud Machine Learning Engine owned by Google in order to improve the system of classification items of the company for the searching, tracking and observation of items to be encountered by a ship in the sea [16].

\subsection{3 Aviation}

The last years, the airlines have access to more data than they had in the past. What they need now is to combine and analyze those data appropriately. Machine learning has contributed and the results are impressive. The Emirates airline has recently announced the commencement of fiveyear cooperation with the University of Oxford in order to create a scientific lab of data. With the participation of a team of scientists, engineers, social scientists and domain experts from the Emirates and the Oxford University, the Oxford University Data Science Lab will be seeking new ways of predicting the request and improving availability of seats in the whole airline network, offering instant and tangible benefits to the Emirates Company. It is going to make use of the data, the machine learning, Maths and the big data in order to rationalize the processes of the Company. Becoming part of a new business strategy that the Company has recently presented, the team will be trying and developing new business solutions utilizing big data and data analysis in real time, contributing to the further transformation of the Company to a customer centered one in travelling experience [17].

\subsection{Railway lines}

The machine learning is a field of science that provides many of the capacities needed in the Technical Strategy of Railway lines, but it still remains low in the railway industry. The limited solutions that are really developed in the railway network attribute great benefits in the railway businesses and the passengers.

The railway businesses is a sector which performance depends more and more on their abilities to draw knowledge from complex groups of data and make the optimum decisions in real time. The Machine Learning has the ability to improve the business's performance by increasing the level of efficiency in making decisions and total procedures.

The efficient management of information and data is of vital importance for the railways, a closely conjugated system of programmes the changes of which anywhere can have critical consequences on their functionality [18].

Machine learning, as a new approach on the operational performance of railways, constitutes therefore an interesting idea, disputing the traditional ways and procedures which were reliable in the past but now have started to reach their limits. The railway lines in order to be competitive into a rapidly changing industry, they have to focus on a number of basic challenges. When it comes to operational performances in the railway section, the capacity to decrease and predict delays of the trains is definitely its main indicator.

1. In 2016, the German company ICT of Fujitsu, in collaboration with SRI International which resides in California, created an application to predict the time delay of a train, using machine learning. The application learns from previous delays, it combines them with previous operational data and it consequently makes precise predictions of delays based on machine learning. This can later appear to the passenger through websites and mobile phones applications and it can have potential benefits on any management and recovery of services which depend of delay predictions. 
2. The Indian railway company Rail Yatri, constructed an algorithm of prediction using techniques of the machine learning and statistical modelling techniques to predict the time arrival of trains. The system is trained by history data and it can offer the passengers realistic estimated time for the arrival of the trains. The learning algorithm of the Rail Yatri Company bears in mind other parameters like the increase of traffic, the speed, the condition of the railway line etc. and adjusts itself, rendering the predictions better through the timeline of the company. It uses clustering techniques for the planning of the trains in thousands of patterns to make an improved prediction in real time.

3. In the United Kingdom, four feasibility studies are funded for railway transportations the result of the research made by the organization Rail Safety and Standards Board (RSSB) entitled "Data Sandbox" and refers to the improvement of the performance of the railway network using technologies of machine learning and tools of data analysis. The feasibility studies (which are about to be completed in March 2019) show their abilities, to predict delays and to inform the time arrivals of a train in the station. The present networks of Network Rail were used in order to make the original models and the facts of network operators of the railways in order to observe the impact that could have been caused by changes in the transportation. The tools that are shaped in the research "Data Sandbox" can be used for the control of interventions in delays scenarios in order to produce emergency plans and improve the passengers' experience [19].

In reality, machine learning is in the centre of every "smart" adaptive system that we can think of and it already offers massive benefits on the railway businesses. Whatever produces data, can potentially aim in machine learning, revealing its importance and usefulness as a future technology. The Machine Learning can offer the keys to the solution of complicated railway problems which can come from the efficient management of the equipment, the planning and programming of services as well as the implementation of the observation plans for the credibility and the availability of the railway network.

The Railways in Greece is about a century and a half old, throughout this period of time there have been many changes, the state grew bigger and the railway network expanded. Many times the Railway was reorganized in order to cover the bigger and complex needs for transport of the Greek society. Nowadays the technology has penetrated every single aspect of our everyday life. It would be unthinkable to exclude the Greek Railways from modernization [20]. Time delays in route performance and postponements of the necessary works on the railway network and the equipment should not be an option. The Greek railway has always been and needs to keep having the leading role in the productive reconstruction of the country and its fair growth. The applications of Machine Learning can successfully confront the new challenges which will multiply the railway flow and will make the railway sector financially successful.

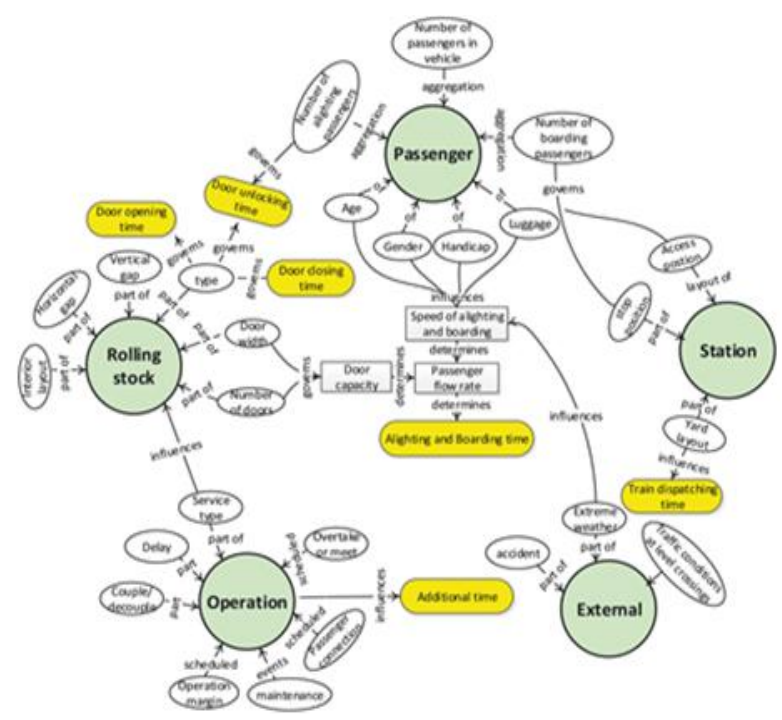

Fig 3: Machine learning application in railway

\section{PROBLEMS OF GREEK RAILWAYS}

The railway transportation in Greece are available in four rail rangesand their services are managed by different companies and organizations. Its main target is the transportation of citizens with safety, reliability, comfort passenger-centered philosophy and respect to the environment caring at the same time for the constant improvement of the quality of the services offered.

The future and the success of every railway company is defined by their ability to assess and make use of their most valuable sources, the business knowledge. The management of every company has to be effectiveness. Informational Systems in order to improve their competitive position, reducing the costs and improving their services. It is of great importance for every company let alone for a transportation company to furtherly develop their "smart" systems and to adopt smart tools like machine learning [21].

One of the components of safe and reliable facility of the railway system is the Maintenance of Rolling Stock which, by nature, is internationally the most costly department, as far as expenses is concerned. It is, at the same time, the most vulnerable too, as their negligent performance can consequently lead to the decrease of reliability up to the cause of devastating disasters i.e. huge material damage and loss of lives. The Rolling Stock is one of the four subsystems of structural nature (infrastructure, energy, control - separation and signaling rolling stock) in the railway system. The maintenance of the rolling stock belongs to one of the three sectors of functional nature or the railways systems (business operation and traffic management, maintenance, telematics applications). The main and of specific nature demands that are arisen for the rolling stock concern their credibility and availability, the technical compatibility and control.

A series of problems that the Management of Maintenance of Rolling Stock faces is the task assignment to the technical teams experientially. One critical disadvantage is calculation difficulty and monitoring the cost of works of maintenance because of lack of cohesive approach among the depots as far as the coding of maintenance and damage tasks is concerned. The handwritten keeping of a great number of data limits the ability for reliable prediction of consumption of spare parts / materials having as a result the increase of time of the trains not available and decrease of the time life of the rolling stock. 
The creation of a model of machine learning for the prediction and the data mining is in position to be the basic tool in the improvement in the process of making decisions by the management in order to obtain a better planning and effective management of the train traffic [22].

On the railway lines there is a high level of congestion because of the great number of trains carrying either passengers or goods and the highly limited infrastructure. Very often negative effects can be caused to the whole railway network by a relatively unimportant incident like a delayed crew or by an important incident like a defected train causing many delays to a big number of trains and consequently the inconvenience of the passengers and the delay of goods. The delay is usually caused by a single train that is able though to cause a chain of problems to the other trains in the railway network.

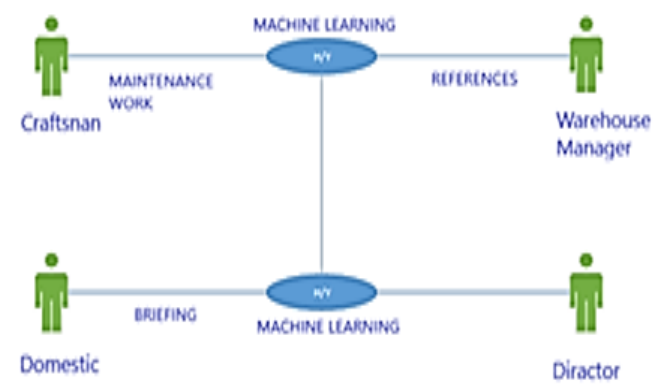

\section{Fig 4: UML Chart on New Procedures Adopted in Rolling Stock Management}

A necessary requirement for the smooth functioning of the railway network is the avoidance of train halt on the main passenger's track which can cause traffic interruption leading to great delays in the carrying out of the timetable and of course it challenges the greatest target of railways which is the safety of passengers and goods [23].

The model of machine learning will significantly help on the planning and improvement of the trains' maintenance, the effective detection of damages and the simplification of planning about the train traffic.

Many important procedures of the majority of the Greek industries are caused by personal effort. Some of the tasks are difficult and time consuming, though. A safe way to solve technical problems is the broad use of computers. The problem of planning the railway services is one of them. The traffic observation, the vehicle location, the danger analysis in real time are problems of improving services which are demanding and can be constantly improved with new techniques. The lack of implementation of observation plan for the credibility and availability of the equipment under construction, the everyday preparation of the trains before the beginning of their schedule on the rails and the responsibility for the application of the maintenance programmes of the rolling stock and the corresponding booklets - task instructions are the obstacles that do not let the Greek railway rolling stock to develop and to improve their productive services to the passenger [24]. They are usually blocked by the balance between the cost, the time and the performance. The ability of the computer to be used when there are great quantities of stored information constitute the machine learning a very good choice that will provide the guidance for the solution of problems and making proper decisions. A smart and nonstop system of machine learning will offer considerable benefits for the solution of more problems and the achievement of better and faster business goals.

\section{CONCLUSIONS}

The sector of machine learning is involved in all the processes that give computers capacities more complex than the undertaking of simple and planned processes. It uses computing methods and algorithms in order to construct systems able to learn. The future and the success of every business is defined by their capacity to assess its most valuable source, the business knowledge. The business environment is getting more and more complicated with the business units to demand complex data for the undertaking of all their functions. The railway businesses is a sector which performance depends more and more on their ability to draw knowledge from complex units of data and make the best decisions in real time. The railway industry produces and collects daily a huge amount of information, from multiple sources and in various forms.

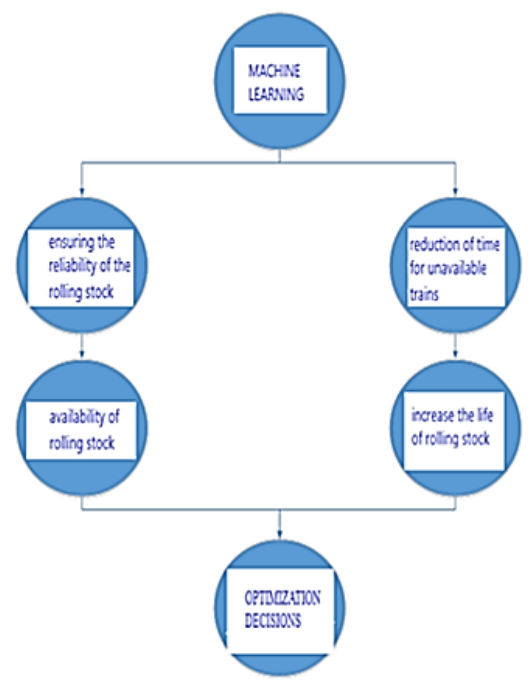

Fig 5: Flow chart for machine learning applications to rolling stock

The machine learning applications ensure the most appropriate management of the growing amount of information and data, improving their effectiveness in making decisions. Using machine learning applications every railway company implements a dynamic management of the trains' and networks maintenance processes resulting in significant saving sources and working hours. The decrease of the total cost on maintenance can reach $40 \%$ with the elimination of the extra working hours and the purchase of inefficient spare parts. The use of machine learning methods offers to every railway company the ability to analyze data coming from sensors and to observe from distance the "attitude" of the equipment aiming to the prediction, diagnosis and the correction of faults. It also gives the advantage of utilizing real time information coming from the same machines and their systems, improving directly the services offered to the passengers. It combines productivity, profit and high quality services with high standard of social and environmental responsibility. The results given offer to the managers to utilize to its peak the qualifications of the human and material resources of the company succeeding their aims to provide services of high standard. To sum up, the capacity given for the development of innovative programmes and techniques promoting new ways of improvement and upgrading of the 
relationship between the company and the passengers is highly significant.

The creation of a machine learning model for the prediction and knowledge mining is in position to constitute the basic tool for the improvement of the process of making decisions from the management of every railway company, aiming to the better planning and the more effective management of the train traffic. As it is proved in practice, the digital transformation of a traditional railway company does not have positive effects only in their balance, but also in the travelling experience of their passengers-customers, as the delays and the inconvenience are diminished, while the most important target is accomplished, the safety of the passengers and the goods.

\section{ACKNOWLEDGMENTS}

All authors would like to thank the University of West Attica and specifically the Post Graduate Program of Studies (MSc) "New Technologies in Shipping and Transport", for the financial support provided to them to undertake this research project..

\section{REFERENCES}

[1] M. I. Jordan and T. M. Mitchell, 'Machine learning: Trends, perspectives, and prospects,' Science, Vol. 349, Issue No. 6245, pp. 255-260, (2015).

[2] R. S. Michalski, J. G. Carbonell, and T. M. Mitchell, Machine learning: An artificial intelligence approach. Published by Springer Science \& Business Media, (2013).

[3] Kyrkos, E. . 'Business intelligence and data mining' [eBook] Athens: Hellenic Academic Libraries Link. Chapter 4. Available Online at: http://hdl.handle.net/11419/1231 (2015)

[4] Rajiv Chopra, 'Machine Learning' Published by KHANNA PUPLISHING, (2018).

[5] Stalidis, G., Kardaras, 'Data Management and Business Intelligence.' [eBook] Athens: Hellenic Academic Libraries Link. Chapter 3. Available Online at: http://hdl.handle.net/11419/116 (2015).

[6] J.McCarthy, E. Feigenbaum. 'Arthur Samuel: Pioneer in Machine Learning', AI Magazine, Vol.11, Issue 3 pp.1 (2018)

[7] Olivier Marteaux, entries: 'Machine Learning and its value proposition for the rail industry' Retrieved from https://www.rssb.co.uk/Pages/machine-learning-and-itsvalue-proposition-for-the-rail-industry.aspx

[8] Jagdeep Kaur, 'Feature selection based efficient machine learning technique for email spam predict', International Journal of Engineering Applied Sciences and Technology, Vol.2, Issue 12, ISSN No. 2455 - 2143, pp.13-19, (2018)

[9] Aikaterini Fountoulaki 'Augmenting statistical quality control with machine learning techniques' University of Patras, PhD (2011)

[10] Ioannis Mademlis, Machine learning and computer vision methods for intelligent video analysis, Aristotle University of Thessaloniki, PhD (2018)

[11] I. Devi, G.R. Karpagam, B. Vinoth Kumar, 'A survey of machine learning techniques, Int. J. of Computational Systems Engineering Vol. 3,Issue No.4 pp. 203 - 212. (2017)

[12] Vlahavas, P. Kefalas, N. Bassiliades, F. Kokkoras, I. Sakellariou. 'Artificial Intelligence' - 3rd Edition, ISBN: 978-960-8396-64-7 Published by University of Macedonia Press / Greece, (2011).Fröhlich, B. and Plate, J. 2000. The cubic mouse: a new device for threedimensional input. In Proceedings of the SIGCHI Conference on Human Factors in Computing Systems

[13] Katerina Georgouli, 'Artificial Intelligence' Published by SEAV (2015)

[14] Qiu, Q. Wu, G. Ding, Y. Xu, and S. Feng, 'A survey of machine learning for big data processing,' EURASIP Journal on Advances in Signal Processing, Vol.67 ,Issue No. 1, pp. 1687-6180 ( 2016)

[15] Julie Chao , 'Machine Learning to Help Optimize Traffic and Reduce Pollution' ,Berkeley Institute of Transportation Studies, Retrieved from https://its.berkeley.edu/node/13327

[16] C. Xu, D. Zhang, Z. Zhang, and Z. Feng, 'BgCut: Automatic Ship Detection from UAV Images,' Thee Scientific World Journal, Vol. 2014, Issue No. 171978, (2014)

[17] Peter Grindrod , Coralia Cartis ,Christopher Lester ,Andrew Mellor, Stephen Roberts ,International Workshop 'Network Science meets Matrix Functions'Emirates Colloquium, Oxford-Emirates Data Science Lab, (2016)

[18] A. Thaduri, D. Galar, and U. Kumar, 'Railway assets: a potential domain for big data Analytics' Procedia Computer Science, Vol. 53, pp. 457-467, (2015)

[19] Andy Gosney, Billy Denyer, Robin Foreshew, Rebecca Hackett, International conference: Data Sandbox: Improving Network Performance Competition, Rail Research UK Association (RRUKA) (2017)

[20] C. Turner, A. Tiwari, A. Starr, and K. Blacktop, 'A review of key planning and scheduling in the rail industry in Europe and UK,' Proceedings of the Institution of Mechanical Engineers, Part F: Journal of Rail and Rapid Transit, Vol. 230, Issue No. 3, pp. 984 998(2016)

[21] Bliemer M, Raadsen M, Brederode L, Bell M, Wismans $\mathrm{L}$, and Smith $\mathrm{M}$ 'Genetics of traffic assignment models for strategic transport planning' Transport Reviews, Vol.37 Issue No 1,pp.56-78.(2017)

[22] Lu Dai 'A machine learning approach for optimization in railway planning, PhD Delft University of Technology, (2018)

[23] Lindfeld A., 'Railway capacity analysis: Methods for simulation and evaluation of timetables, delays and infrastructure' Stockholm: KTH Royal Institute of Technology PhD Thesis (2015)

[24] Hongfei Li, Dhaivat Parikh , Qing He , Buyue Qian , Zhiguo Li , Dongping Fang, Arun Hampapur 'Improving rail network velocity: A machine learning approach to predictive maintenance' Transportation Research Part C, Vol 45 pp 17-26 (2014). 